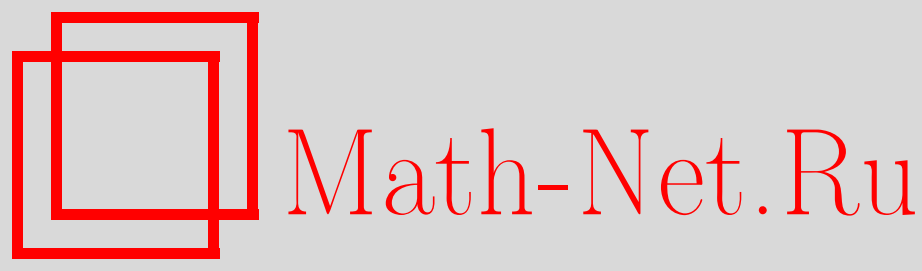

С. С. Марченков, Об элементарных словарных функциях, получаемых на основе ограниченной префиксной конкатенации, Дискрет. матем., 2015, том 27, выпуск $3,44-55$

DOI: https://doi.org/10.4213/dm1334

Использование Общероссийского математического портала Math-Net.Ru подразумевает, что вы прочитали и согласны с пользовательским соглашением http://www.mathnet.ru/rus/agreement

Параметры загрузки:

IP: 54.197 .130 .99

26 апреля 2023 г., 13:27:37

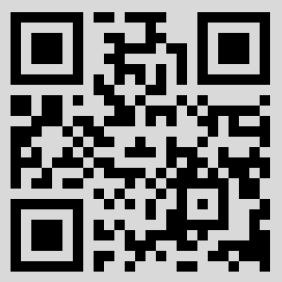




\title{
Об элементарных словарных функциях, получаемых на основе ограниченной префиксной конкатенации
}

\author{
() 2015 г. С. С. Марченков*
}

На множестве словарных функций в алфавите $\{1,2\}$ вводится операция ограниченной префиксной конкатенации. На основе этой операций и операции суперпозиции определяется класс ВРС полиномиально вычислимых функций. Устанавливается принадлежность классу ВРС ряда словарных функций, а также замкнутость класса ВРС относительно некоторых известных операций. Вводится некоторый тип двуленточных нестирающих машин Тьюринга и доказывается, что функции из класса ВРС можно вычислить на машинах этого типа за полиномиальное время.

Работа выполнена при поддержке Российского фонда фундаментальных исследований, проект 13-01-00958.

Ключевые слова: операция ограниченной префиксной конкатенации, полиномиально вычислимая функция.

\section{1. Введение}

За последние 70 лет в теории рекурсивных функций введено несколько классов так называемых элементарных функций. Понятие «элементарная рекурсивная функция» не формализовано, и различные авторы подошли к введению этого понятия с разных позиций. Общим здесь, по-видимому, является то, что определяемые классы составляют сравнительно небольшую часть класса примитивно рекурсивных функций, содержат наиболее употребительные арифметические функции, являются перечислимыми и имеют, как правило, индуктивные определения с использованием некоторых «ограниченных» операций. Типичными примерами являются класс функций, элементарных по Кальмару [5, 4, 2], и класс функций, элементарных по Сколему [6, 2].

Отметим, что процесс определения новых классов элементарных функций продолжается, и одна из тенденций в этом направлении, на наш взгляд, состоит в раскрытии понятия полиномиально вычислимой функции. Сам класс $P$ полиномиально вычислимых функций задается в терминах вычислений на машинах Тьюринга, однако его определение нетрудно сделать индуктивным (см., например, [3]). Для

*Место работы: МГУ им. М. В. Ломоносова, e-mail: ssmarchen@yandex.ru 
некоторых приложений класс $P$ оказывается слишком большим (функции с полиномиально растущей длиной двоичной записи значений имеют сверхполиномиальный рост).

Другой подход в этом направлении - определить чисто синтаксическим образом некоторый класс словарных функций, часто используемых в приложениях, и затем доказать полиномиальную вычислимость функций этого класса и, возможно, совпадение (несовпадение) данного класса с классом $P$. Наиболее распространенной словарной функцией является конкатенация, а наиболее распространенной операцией порождения функций - суперпозиция. В связи с этим представляется естественным определить некоторый класс словарных функций на основе конкатенации и суперпозиции. Однако в этом случае при выборе сравнительно простых исходных функций такой класс оказывается слишком бедным.

Выход из положения здесь может состоять в том, чтобы на базе конкатенации определить некоторую «ограниченную» словарную операцию, которая позволит существенно расширить класс определимых функций. Вместе с тем эта операция не должна выводить за пределы класса полиномиально вычислимых функций. В качестве такой операции мы предлагаем операцию ограниченной префиксной конкатенации (сокращенно ВРС). На основе операций ВРС и суперпозиции мы определяем класс полиномиально вычислимых словарных функций, который также имеет обозначение ВРС. Мы доказываем принадлежность классу ВРС ряда конкретных словарных функций, а также замкнутость класса ВРС относительно некоторых известных операций. В качестве следствия в класс ВРС «погружается» класс ограниченно арифметических предикатов А. В. Кузнецова $[1,2]$, рассматриваемых от длин словарных переменных. Кроме того, мы определяем некоторый тип двуленточных нестирающих машин Тьюринга и доказываем, что функции из класса ВРС вычислимы на машинах этого типа.

\section{2. Основные понятия}

Рассмотрим алфавит $\Sigma=\{1,2\}$ и множество $\Sigma^{*}$ всех конечных слов в этом алфавите, включая пустое слово $\Lambda$. Определим ряд словарных функций на множестве $\Sigma^{*}$. Основной словарной функцией будет конкатенация $\operatorname{con}\left(x_{1}, x_{2}\right)$, которую будем обозначать $x_{1} * x_{2}$ (или даже $x_{1} x_{2}$ ). Слово $x_{1} * x_{2}$ получается из слова $x_{1}$ приписыванием справа слова $x_{2}$. При этом, очевидно, $\Lambda * x=x * \Lambda=x$. Длину слова $x$ обозначаем через $|x|$, считая $|\Lambda|=0$. Для задания периодических слов применяем степенные обозначения: например, $1^{n}$ есть слово, состоящее из $n$ единиц. Пусть $I$ обозначает множество всех селекторных функций $I_{i}^{n}(1 \leqslant i \leqslant n, n=1,2, \ldots)$, где для любых слов $x_{1}, \ldots, x_{n}$ из $\Sigma^{*}$ имеем $I_{i}^{n}\left(x_{1}, \ldots, x_{i}, \ldots, x_{n}\right)=x_{i}$. Положим

$$
s_{1}(x)=x * 1, \quad s_{2}(x)=x * 2 .
$$

Словарную функцию subtr определим соотношениями

$$
\operatorname{subtr}\left(x_{1}, x_{2}\right)=\left\{\begin{array}{l}
y, \text { если } y-\text { такое (единственное) слово, что } y * x_{2}=x_{1}, \\
\Lambda \text { в остальных случаях. }
\end{array}\right.
$$

Для функции $\operatorname{subtr}\left(x_{1}, x_{2}\right)$ будем также использовать обозначение $x_{1} \div x_{2}$. Легко видеть, что при любом $x$ имеем $x \div x=\Lambda$. 
Говорим, что слово $x$ есть префикс (начало) слова $y$, если для некоторого слова $z$ (возможно, пустого или равного слову $y$ ) выполняется соотношение $y=x * z$. Бинарное отношение «слово $x$ есть префикс слова $y$ » обозначем в виде $x \sqsubseteq y$. На основе функции конкатенации определяем операцию ограниченной префиксной конкатенации:

$$
f\left(x_{1}, \ldots, x_{n}, y\right)=\underset{z \sqsubseteq y}{\operatorname{Con}} g\left(x_{1}, \ldots, x_{n}, z\right),
$$

где правая часть равенства является сокращением для выражения

$$
g\left(x_{1}, \ldots, x_{n}, \Lambda\right) * g\left(x_{1}, \ldots, x_{n}, z_{1}\right) * \ldots * g\left(x_{1}, \ldots, x_{n}, z_{l}\right),
$$

$l=|y|, z_{1}, \ldots, z_{l}-$ все непустые префиксы слова $y$ в порядке возрастания их длин и $z_{l}=y$.

Обозначим через ВРС класс всех функций, которые можно получить из исходных функций

$$
s_{1}, s_{2}, \quad \text { con, subtr, } I
$$

с помощью операций суперпозиции и ограниченной префиксной конкатенации.

Введем еще одну «ограниченную» операцию - операцию ограниченной минимизации. Если $f\left(x_{1}, \ldots, x_{n}\right)$ - функция на множестве $\Sigma^{*}$, то пусть $(\mu z)_{z \sqsubseteq y}\left(f\left(x_{1}, \ldots, x_{n-1}, z\right)=x_{n}\right)$ равно наименьшему (по длине) префиксу слова $x_{n}$, для которого выполняется равенство $f\left(x_{1}, \ldots, x_{n-1}, z\right)=x_{n}$, если такой префикс существует, и равно $\Lambda$ в противном случае.

Пусть $\rho\left(x_{1}, \ldots, x_{n-1}, z\right)$ - предикат на множестве $\Sigma^{*}$. Посредством $(\exists z)_{z \sqsubseteq y} \rho\left(x_{1}, \ldots, x_{n-1}, z\right)$ обозначим предикат (от переменных $x_{1}, \ldots, x_{n-1}, y$ ), который совпадает с предикатом

$$
\rho\left(x_{1}, \ldots, x_{n-1}, \Lambda\right) \vee \rho\left(x_{1}, \ldots, x_{n-1}, z_{1}\right) \vee \ldots \vee \rho\left(x_{1}, \ldots, x_{n-1}, z_{l}\right),
$$

где $z_{1}, \ldots, z_{l}$ - все непустые префиксы слова $y$. Аналогичным образом определим предикат $(\forall z)_{z \sqsubseteq y} \rho\left(x_{1}, \ldots, x_{n-1}, z\right)$. Операции перехода от предиката $\rho$ к определенным выше предикатам с ограниченными кванторами будем называть операциями ограниченной префиксной квантификации.

\section{3. Класс ВРС}

Установим, прежде всего, принадлежность классу ВРС некоторых простых словарных функций. Как отмечалось, функция-константа $\Lambda$ определяется с помощью формулы $\operatorname{subtr}(x, x)$. Остальные функции-константы можно получить из константы $\Lambda$ с помощью функций $s_{1}$ и $s_{2}$. Положим

$$
\begin{aligned}
& \operatorname{sg}(x)= \begin{cases}\Lambda, & \text { если } x=\Lambda, \\
1 & \text { в противном случае, }\end{cases} \\
& \overline{\operatorname{sg}}(x)= \begin{cases}1, & \text { если } x=\Lambda, \\
\Lambda & \text { в противном случае. }\end{cases}
\end{aligned}
$$


Имеем $\overline{\operatorname{sg}}(x)=1 \div x, \operatorname{sg}(x)=\overline{\operatorname{sg}}(\overline{\operatorname{sg}}(x))$. Нетрудно видеть, что применение операции $\mathrm{Con}_{z \sqsubseteq y}$ к функции $I_{1}^{2}(x, z)$ дает функцию $x^{|y|+1}$. Далее получаем функцию $x^{|y|}$ : $x^{|y|}=x^{|y|+1} \div x(|y|$-я «степень» $x)$.

Если $\rho\left(x_{1}, \ldots, x_{n}\right)$ - предикат на множестве $\Sigma^{*}$, то характеристической функиией предиката $\rho$ называем такую функцию $\chi_{\rho}\left(x_{1}, \ldots, x_{n}\right)$, которая принимает лишь значения $\Lambda, 1$ и при этом предикат $\rho$ совпадает с предикатом $\chi_{\rho}\left(x_{1}, \ldots, x_{n}\right)=1$. Обозначим через $\mathrm{BPC}_{*}$ класс всех предикатов, характеристические функции которых принадлежат классу ВРС (предикаты класса ВРС).

Предикаты $|x|=|y|$ и $|x|<|y|$ принадлежат классу ВРС. Это сразу следует из вида их характеристических функций: $\overline{\operatorname{sg}}\left(\left(1^{|x|} \div 1^{|y|}\right) *\left(1^{|y|} \div 1^{|x|}\right)\right)$ и $\operatorname{sg}\left(1^{|y|} \div 1^{|x|}\right)$.

Утверждение 1. Класс $\mathrm{BPC}_{*}$ замкнут относительно операций логики высказываний, подстановки констант (из множества $\Sigma^{*}$ ), перестановки и отождествления переменных, а также относительно операций ограниченной пребиксной квантификации.

Доказательство. Пусть $\rho(\tilde{x}), \sigma(\tilde{x})-$ предикаты класса $\mathrm{BPC}_{*}, \chi_{\rho}(\tilde{x}), \chi_{\sigma}(\tilde{x})-$ их характеристические функции. Тогда характеристическими функциями предикатов $\bar{\rho}(\tilde{x})$ и $\rho(\tilde{x}) \vee \sigma(\tilde{x})$ будут функции

$$
\overline{\operatorname{sg}}(\rho(\tilde{x})), \quad \operatorname{sg}\left(\chi_{\rho}(\tilde{x}) * \chi_{\sigma}(\tilde{x})\right) .
$$

Для предиката $(\exists z)_{z \sqsubseteq y} \rho\left(x_{1}, \ldots, x_{n-1}, z\right)$, полученного из предиката $\rho$ с помощью операции навешивания ограниченного квантора существования, характеристической функцией будет функция $\operatorname{sg}\left(\operatorname{Con}_{z \sqsubseteq y} \chi_{\rho}\left(x_{1}, \ldots, x_{n-1}, z\right)\right)$. Замкнутость класса $\mathrm{BPC}_{*}$ относительно остальных операций очевидна. Утверждение доказано.

Покажем, что классу $\mathrm{BPC}_{*}$ принадлежат предикаты $x=y$ и $x \neq y$. Ввиду доказанного утверждения можно ограничиться только предикатом $x=y$. Однако $x=y$ тогда и только тогда, когда $|x|=|y|$ и функция $(1 * x) \div y$ принимает значение 1 . Поскольку при условии $|x|=|y|$ она принимает лишь значения 1 и $\Lambda$, для последнего предиката можно рассмотреть характеристическую функцию $\operatorname{sg}((1 * x) \div y)$.

Утверждение 2. Класс ВРС замкнут относителъно операции ограниченной минимизаиии.

Доказательство. Пусть

$$
f\left(x_{1}, \ldots, x_{n}, y\right)=(\mu z)_{z \sqsubseteq y}\left(g\left(x_{1}, \ldots, x_{n-1}, z\right)=x_{n}\right)
$$

и $g \in \mathrm{BPC.} \mathrm{Введем} \mathrm{предикат} \rho\left(x_{1}, \ldots, x_{n}, z\right)$, истинный в том и только том случае, когда $z$ есть решение уравнения $g\left(x_{1}, \ldots, x_{n-1}, z\right)=x_{n}$ и никакое начало слова $z$, отличное от слова $z$, решением этого уравнения не является. Принадлежность предиката $\rho$ классу $\mathrm{BPC}_{*}$ вытекает из первой части утверждения 1 и эквивалентности

$\rho\left(x_{1}, \ldots, x_{n}, z\right) \equiv\left(g\left(x_{1}, \ldots, x_{n-1}, z\right)=x_{n}\right) \&(\forall v)_{v \sqsubseteq z}((v \neq z) \Rightarrow$

$$
\left.\left(g\left(x_{1}, \ldots, x_{n-1}, z\right) \neq x_{n}\right)\right) .
$$


Пусть $\chi$ - характеристическая функция предиката $\rho$. Тогда будем иметь

$$
f\left(x_{1}, \ldots, x_{n}, y\right)=\operatorname{Con}_{z \sqsubseteq y} z^{\chi\left(x_{1}, \ldots, x_{n}, z\right)} .
$$

Утверждение доказано.

Рассмотрим предикаты

$B(x, y) \equiv($ слово $x$ есть начало слова $y)$,

$E(x, y) \equiv($ слово $x$ есть конец слова $y)$,

$C(x, y) \equiv($ слово $x$ входит в слово $y)$.

Покажем, что они принадлежат классу $\mathrm{BPC}_{*}$. Для предикатов $B$ и $E$ это следует из эквивалентностей

$$
B(x, y) \equiv(\exists z)_{z \sqsubseteq y}(z=x), \quad E(x, y) \equiv(\exists z)_{z \sqsubseteq y}(z * x=y),
$$

для предиката $C$ - из эквивалентности

$$
C(x, y) \equiv\left(\exists z_{1}\right)_{z_{1} \sqsubseteq y}\left(\exists z_{2}\right)_{z_{2} \sqsubseteq y}\left(z_{1}=z_{2} * x\right) .
$$

Пусть $\rho_{1}(\tilde{x}), \ldots, \rho_{1}(\tilde{x})$ - попарно несовместные предикаты. Будем говорить, что функция $f(\tilde{x})$ получена из функций $g_{1}(\tilde{x}), \ldots, g_{m}(\tilde{x}), g_{m+1}(\tilde{x})$ с помощью операции разбора случаев по предикатам $\rho_{1}, \ldots, \rho_{m}$, если

$$
f(\tilde{x})=\left\{\begin{array}{l}
g_{1}(\tilde{x}), \quad \text { если } \rho_{1}(\tilde{x}), \\
\ldots \ldots \ldots \ldots \ldots \ldots \ldots \\
g_{m}(\tilde{x}), \quad \text { если } \rho_{m}(\tilde{x}), \\
g_{m+1}(\tilde{x}) \text { в остальных случаях. }
\end{array}\right.
$$

Утверждение 3. Класс ВРС замкнут относительно операщии разбора случаев по предикатам.

Доказательство. Пусть функции $g_{1}, \ldots, g_{m+1}$ принадлежат классу ВРС, а предикаты $\rho_{1}, \ldots, \rho_{m}-$ классу $\mathrm{BPC}_{*}$. И пусть функция $f(\tilde{x})$ получается из функций $g_{1}, \ldots, g_{m+1}$ и предикатов $\rho_{1}, \ldots, \rho_{m}$ согласно формуле $(3)$. Покажем, что функция $f(\tilde{x})$ также принадлежит классу ВРС. Обозначим через $\chi_{1}, \ldots, \chi_{m}$ характеристические функции предикатов $\rho_{1}, \ldots, \rho_{m}$. Тогда

$$
f(\tilde{x})=\left(g_{1}(\tilde{x})\right)^{\chi_{1}(\tilde{x})} * \ldots *\left(g_{m}(\tilde{x})\right)^{\chi_{m}(\tilde{x})} *\left(g_{m+1}(\tilde{x})\right)^{\chi(\tilde{x})},
$$

где $\chi(\tilde{x})=\overline{\operatorname{sg}}\left(\chi_{1}(\tilde{x}) * \ldots * \chi_{m}(\tilde{x})\right)$. Утверждение доказано.

Обозначим через first $(x), \operatorname{last}(x)$ функции, которые выдают первый (последний) символы слова $x$, если слово $x$ непусто, и $\Lambda$ в противном случае. Используя утверждение 3, устанавливаем принадлежность функции first классу BPC:

$$
\operatorname{first}(x)= \begin{cases}1, & \text { если } B(1, x), \\ 2, & \text { если } B(2, x), \\ \Lambda & \text { если } x=\Lambda\end{cases}
$$

Аналогично доказывается принадлежность классу BPC функции last. 
Пусть $\operatorname{pref}_{|y|}(x)$ равно префиксу слова $x$ длины $|y|$, если $|y| \leqslant|x|$, и равно $\Lambda$ при $|y|>|x|$. Имеем

$$
\operatorname{pref}_{|y|}(x)=\operatorname{Con}_{z \sqsubseteq x} z^{\chi(y, z)},
$$

где $\chi(y, z)$ есть характеристическая функция предиката $|y|=|z|$. Таким образом, функция $\operatorname{pref}_{|y|}(x)$ принадлежит классу ВРС.

Обозначим через $\operatorname{inv}(x)$ функцию, которая дает инверсию слова $x$ (т.е. слово, составленное из символов слова $x$, записанных в обратном порядке). Следующая формула устанавливает принадлежность функции inv классу ВРC:

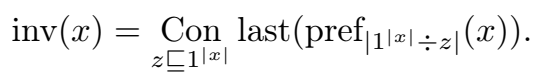

По аналогии с операцией ограниченной префиксной конкатенации введем операцию ограниченной суффиксной конкатенации, для которой будем использовать обозначение $\operatorname{Con}_{y \sqsupseteq z}$. Действие операции $\operatorname{Con}_{y \sqsupseteq z}$ на функцию $g(\tilde{x}, z)$ состоит в образовании конкатенации слов $g\left(\tilde{x}, z_{l}\right), \ldots, g\left(\tilde{x}, z_{1}\right), g(\tilde{x}, \Lambda)$, где $z_{1}, \ldots, z_{l}$ - все непустые суффиксы слова $y$, выписанные в порядке возрастания длин.

Утверждение 4. Класс ВРС замкнут относительно операции ограниченной суффиксной конкатенации.

Доказательство. Очевидно, что при инверсии слова суффиксы переходят в префиксы и обратно. Поэтому

$$
\underset{y \sqsupseteq z}{\operatorname{Con}} g(\tilde{x}, z)=\operatorname{inv}(\underset{z \sqsubseteq \operatorname{inv}(y)}{\operatorname{Con}} g(\tilde{x}, z)) .
$$

Утверждение доказано.

Нетрудно видеть, что операции ограниченной префиксной конкатенации и ограниченной суффиксной конкатенации «двойственны» друг другу относительно преобразования inv. Поэтому ровно такой же класс ВРС получится, если вместо операции ограниченной префиксной конкатенации рассмотреть операцию ограниченной суффиксной конкатенации, а вместо исходных функций $s_{1}, s_{2}$, subtr взять соответствующие «двойственные» функции.

Класс ВРС будет замкнут относительно еще двух операций, определенных на основе ограниченной префиксной конкатенации: когда рассматриваются префиксы слова $y$, но конкатенация проводится в обратном порядке (начиная со слова $z_{l}=y$ ), и когда рассматриваются суффиксы слова $y$, но конкатенация проводится в порядке возрастания длин суффиксов.

Еще один вариант операции ограниченной конкатенации можно определить на основе всех подслов «ограничивающего» слова $y$. Однако здесь возникают некоторые (но преодолимые) технические трудности, связанные с учетом вхождений одного и того же подслова в слово $y$. Мы не будем останавливаться на детальном определении данного понятия, отметив только, что для операций ограниченной квантификации эти трудности по существу не возникают.

Для классов функций, замкнутых относительно операции суперпозиции, важным свойством является наличие в классе функции, нумерующей пары, и «обратных» к 
ней функций. Мы решим эту задачу для класса ВРС, определив соответствующие функции pair, left и right. С этой целью введем ряд словарных функций и докажем их принадлежность классу ВРС.

Пусть $\operatorname{doub}(x)$ - функция, удваивающая каждый символ слова $x$. Имеем

$$
\operatorname{doub}(x)=\underset{z \sqsupseteq x}{\operatorname{Con}}(\operatorname{first}(z) * \operatorname{first}(z)) .
$$

Нумерационную функцию раir определим равенством

$$
\operatorname{pair}\left(x_{1}, x_{2}\right)=\operatorname{doub}\left(x_{1}\right) * 12 * \operatorname{doub}\left(x_{2}\right) .
$$

Пусть, далее, even $(x), \operatorname{odd}(x)$ - предикаты, определяющие четность (нечетность) длины слова $x$. Для предиката even справедлива эквивалентность

$$
\operatorname{even}(x) \equiv(\exists z)_{z \sqsubseteq x}\left(1^{|x|}=1^{|z|} * 1^{|z|}\right) .
$$

Аналогичная эквивалентность справедлива для предикта odd.

Обозначим через $\operatorname{comress}(x)$ функцию, которая удовлетворяет тождеству $\operatorname{compress}(\operatorname{doub}(x))=x$. Для функции compress получаем выражение

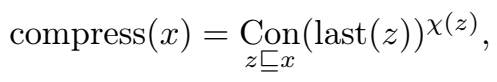

где $\chi(z)$ - характеристическая функция предиката odd.

Пусть $\operatorname{left}^{\prime}(x)$ - функция, которая по слову $x$ вида $\operatorname{pair}\left(x_{1}, x_{2}\right)$ выдает слово $\operatorname{doub}\left(x_{1}\right)$. Имеем

$$
\operatorname{left}^{\prime}(x)=\operatorname{Con}_{z \sqsubseteq x} z^{\chi(x, z)},
$$

где $\chi(x, z)$ - характеристическая функция предиката

$$
\operatorname{even}(z) \& B(z 12, x) \text {. }
$$

Теперь получаем

$$
\operatorname{left}(x)=\operatorname{compress}\left(\operatorname{left}^{\prime}(x)\right) .
$$

Функцию right также получаем в два этапа:

$$
\operatorname{right}^{\prime}(x)=\operatorname{inv}\left(\operatorname{inv}(x) \div\left(21 * \operatorname{inv}\left(\operatorname{left}^{\prime}(x)\right)\right)\right), \quad \operatorname{right}(x)=\operatorname{compress}\left(\operatorname{right}^{\prime}(x)\right) .
$$

Пусть $N=\{0,1, \ldots\}$ - множество целых неотрицательных чисел. Рассмотрим предикаты на множестве $N$ и введем понятие ограниченно арифметического предиката. Предикаты $x+y=z$ и $x \cdot y=z$ считаем ограниченно арифметическими. Из данных предикатов все остальные ограниченно арифметические предикаты получаются с помощью операций логики высказываний, подстановки констант (из множества $N)$, перестановки и отождествления переменных, а также с помощью операций ограниченной квантификации. При этом ограничения в последних операциях имеют вид $z \leqslant y$. Множество всех ограниченно арифметических предикатов обозначим через ВА.

Как отмечалось, функции класса ВРС вычислимы за полиномиальное время. Это является «оценкой сверху» для сложности вычисления данных функций. Однако 
какой-либо аналогичной «оценки снизу» пока не известно. Вместе с тем утверждение 1 (особенно в его второй части) указывает на то, что в классе $\mathrm{BPC}_{*}$ должны содержаться некоторые известные подклассы словарных либо арифметических предикатов. Мы укажем только один из таких подклассов, который связан с классом ограниченно арифметических предикатов А. В. Кузнецова. Именно будем рассматривать предикаты из класса $\mathrm{BPC}_{*}$ только на словах вида $1^{n}$. Формально следует «ограничить» все переменные, используемые в определении таких предикатов, предикатом $1(x)$, который выделяет слова указанного вида. Этот предикат можно задать формулой $1 * x=x * 1$. Тогда сложность вычисления ограниченно арифметических предикатов (но от длин слов) будет являться некоторой «оценкой снизу» для класса предикатов $\mathrm{BPC}_{*}$, поскольку известно [2], что ограниченно арифметическими предикатами можно «моделировать» некоторые вычисления на машинах Тьюринга.

\section{4. Двуленточные машины Тьюринга}

\section{и класс $C$}

Будем рассматривать вариант двуленточных нестирающих машин Тьюринга с ленточным алфавитом $\{0,1,2\}$, входной лентой, имеющей несколько (только читающих) головок, и выходной лентой, предназначенной только для записи результата вычисления. Пусть $\mathcal{T}-$ машина Тьюринга этого типа, имеющая $k$ головок на входной ленте и состояния $q_{1}, \ldots, q_{r}$. Состояние $q_{1}$ считаем начальным состоянием, состояние $q_{r}$ - заключительным. Программа машины $\mathcal{T}$ состоит из команд вида

$$
a_{1} \ldots a_{k} q_{i} \rightarrow b D_{1} \ldots D_{k} q_{j}
$$

где $a_{1}, \ldots, a_{k} \in\{0,1,2\}, b \in\{1,2, \Lambda\}, D_{1}, \ldots, D_{k} \in\{L, R, S\}$ и $i \neq r$. Команда (4) интерпретируется следующим образом. Если в некоторый момент времени машина $\mathcal{T}$ находится в состоянии $q_{i}$ и обозревает $k$ головками на входной ленте символы $a_{1}, \ldots, a_{k}$, то в следующий момент времени она добавит (справа) слово $b$ к имеющемуся на выходной ленте слову, перейдет в состояние $q_{j}$ и совершит $k$ головками одновременно движения в соответствии с символами $D_{1}, \ldots, D_{k}(L-$ сдвиг влево, $R$ - вправо, $S$ - отсутствие сдвига). При этом предполагается, что в начальный момент времени на выходной ленте ничего не записано (записано пустое слово $\Lambda$ ).

Машины рассматриваемого типа предназначены для вычисления словарных функций в алфавите $\{1,2\}$. Чтобы представить на входной ленте набор из $n$ слов $\left(w_{1}, \ldots, w_{n}\right)$ (некоторые из этих слов могут быть пустыми), на ленте записывается слово

$$
0 w_{1} 0 w_{2} 0 \ldots 0 w_{n} 0,
$$

где символ 0 служит разделительным символом, отделяющим соседние слова $w_{i}, \mathrm{a}$ также граничным символом, ограничивающим заданную $n$-ку слов слева и справа. Поскольку мы рассматриваем нестирающие машины Тьюринга, запись (5) будет присутствовать на входной ленте машины в течение всего процесса вычисления.

Условимся, что в начальный момент времени все головки машины располагаются на крайнем левом символе 0 слова (5). Добавим еще одно важное ограничение на 
процесс вычисления: будем рассматривать только такие вычисления, в которых головки не выходят за пределы слова (5) (это условие легко обеспечить за счет соответствующей организации программы машины Тьюринга). Понятно, что при выполнении данных условий машина Тьюринга $\mathcal{T}$ с $k$ головками и $r$ состояниями может работать без зацикливания не более $r l^{k}$ тактов, где $l$ - длина слова (5).

Далее рассматриваем вычисление только всюду определенных словарных функций в алфавите $\{1,2\}$. Будем говорить, что функция $f\left(x_{1}, \ldots, x_{n}\right)$ этого типа вычислима на машине $\mathcal{T}$, если для любого набора $\left(w_{1}, \ldots, w_{n}\right)$ слов в алфавите $\{1,2\}$ машина $\mathcal{T}$, начиная работу со словом (5) на входной ленте, завершает работу в заключительном состоянии и при этом на выходной ленте представлено слово $f\left(w_{1}, \ldots, w_{n}\right)$ (если данное слово пусто, то выходная лента, разумеется, не содержит символов 1,2$)$.

Обозначим через $C$ класс всех словарных функций, вычислимых на определенных выше машинах Тьюринга. Нетрудно убедиться в том, что всякую функцию из класса $C$ можно вычислить на одноленточной машине Тьюринга (с одной головкой на ленте) за полиномиальное время. Однако стоит обратить внимание на то, что если рассматривать, например, вычисление словарных функций из $C$ с конечной областью значений, то необходимый размер ленты можно ограничить линейной функцией (от длины записи аргументов). Для класса $P$ функций, вычислимых за полиномиальное время, до сих пор не известно, возможно ли подобное ограничение размера ленты (для функций с конечной областью значений).

Теорема 1. Имеет место включение $\mathrm{BPC} \subseteq C$.

Доказательство. Легко видеть, что исходные функции класса ВРС принадлежат классу $C$. Поэтому для доказательства теоремы остается проверить, что класс $C$ замкнут относительно операций суперпозиции и ограниченной префиксной конкатенации.

Начнем с операции суперпозиции. Пусть функции $g_{0}, g_{1}, \ldots, g_{m}$ принадлежат классу $C$ и

$$
f\left(x_{1}, \ldots, x_{n}\right)=g_{0}\left(g_{1}\left(x_{1}, \ldots, x_{n}\right), \ldots, g_{m}\left(x_{1}, \ldots, x_{n}\right)\right) .
$$

Предположим также, что $\mathcal{T}_{0}, \mathcal{T}_{1}, \ldots, \mathcal{T}_{m}$ суть машины Тьюринга, которые вычисляют соответственно функции $g_{0}, g_{1}, \ldots, g_{m}$, и машина $\mathcal{T}_{0}$ имеет $k$ головок. Мы опишем, как определить машину Тьюринга $\mathcal{T}$, которая будет вычислять функцию $f$. В определении машины $\mathcal{T}$ будем использовать стандартные технические приемы, применяемые при построении машин Тьюринга.

Прежде всего, для каждой машины $\mathcal{T}_{i}(1 \leqslant i \leqslant m)$ определим три «счетчика» $\mathcal{C}_{i 1}, \mathcal{C}_{i 2}, \mathcal{C}_{i 3}$, т. е. машины Тьюринга (без выходных лент), которые, используя, например, позиционную систему счисления с основанием $l$ (длина слова (5)), с помощью своих головок на входной ленте последовательно «генерируют» натуральные числа, достаточные для нумерации всех моментов работы машины $\mathcal{T}_{i}$. Иными словами, каждая из машин $\mathcal{C}_{i 1}, \mathcal{C}_{i 2}, \mathcal{C}_{i 3}$ может за счет расположения головок на входной ленте «пересчитать» все моменты работы машины $\mathcal{T}_{i}$ вплоть до попадания последней в заключительное состояние. Мы предполагаем также, что процесс «генерирования» чисел посредством счетчиков $\mathcal{C}_{i 1}, \mathcal{C}_{i 2}, \mathcal{C}_{i 3}$ в любой момент можно «обратить», т.е. запустить процедуру прохождения всех ранее достигнутых конфигураций в обратном порядке. 
Основная идея вычисления функции $f$ на машине $\mathcal{T}$ заключается в следующем. Чтобы определить набор $\left(a_{1}, \ldots, a_{k}\right)$ из множества $\{0,1,2\}^{k}$, который воспринимается машиной $\mathcal{T}_{0}$ в некоторый момент времени, машина $\mathcal{T}$ с помощью набора счетчиков $\mathcal{C}_{i 1}, \mathcal{C}_{i 2}, \mathcal{C}_{i 3}$ «запоминает» те моменты времени, в которые символы $\left(a_{1}, \ldots, a_{k}\right)$ впервые появились на выходных лентах машин $\mathcal{T}_{1}, \ldots, \mathcal{T}_{m}$. Чтобы теперь реально восстановить символы $a_{1}, \ldots, a_{k}$, следует до нужного момента времени промоделировать работу подходящих машин $\mathcal{T}_{s}$.

Более подробно, возьмем произвольный набор $\left(w_{1}, \ldots, w_{n}\right)$ слов в алфавите $\{1,2\}$ и положим

$$
z_{1}=g_{1}\left(w_{1}, \ldots, w_{n}\right), \ldots, z_{m}=g_{m}\left(w_{1}, \ldots, w_{n}\right)
$$

Выясним, прежде всего, какие из слов $z_{1}, \ldots, z_{m}$ отличны от пустого слова. Для этого последовательно промоделируем полностью вычисления на машинах $\mathcal{T}_{1}, \ldots, \mathcal{T}_{m}$, отвечающие входному набору $\left(w_{1}, \ldots, w_{n}\right)$. При этом машина $\mathcal{T}$ ничего не выдает на выходную ленту, а лишь определяет пустоту/непустоту слов $z_{1}, \ldots, z_{m}$. Полученную информацию машина $\mathcal{T}$ запоминает за счет внутренних состояний.

Сформулируем теперь основные предположения касательно моделирования вычислений на машине $\mathcal{T}$. Предположим, что в некоторый момент моделирования машина $\mathcal{T}$ определила набор $\left(a_{1}, \ldots, a_{k}\right)$, который воспринимается $k$ головками машины $\mathcal{T}_{0}$ при чтении ею входного слова $0 z_{1} 0 z_{2} 0 \ldots 0 z_{m} 0$, а также состояние, в котором в этот момент находится машина $\mathcal{T}_{0}$. При этом для каждой из $k$ головок машины $\mathcal{T}_{0}$ машине $\mathcal{T}$ известно, на каком именно слове $z_{i}$ располагается данная головка в рассматриваемый момент моделирования (если слово $z_{i}$ пусто, то известно, какой из двух ограничительных символов 0 обозревает данная головка). Более того, предположим, что в случае непустоты слова $z_{i}$ один из счетчиков (будем считать, что это счетчик $\mathcal{C}_{i 1}$ ), приданных этой головке, «хранит» тот момент времени, в который обозреваемый головкой символ впервые появился на выходной ленте машины $\mathcal{T}_{i}$ (два других счетчика в этот момент «хранят» число 0). Предположим, наконец, что к данному моменту моделирования на выходной ленте машины $\mathcal{T}$ имеется ровно то слово, которое в соответствующий момент времени имеется на выходной ленте машины $\mathcal{T}_{0}$.

Покажем, как при сформулированных предположениях получить значения всех рассматриваемых параметров для следующего такта работы машины $\mathcal{T}_{0}$.

Пользуясь найденными набором $\left(a_{1}, \ldots, a_{k}\right)$ и состоянием машины $\mathcal{T}_{0}$ в моделируемый момент времени, машина $\mathcal{T}$ (с помощью программы машины $\left.\mathcal{T}_{0}\right)$ определяет следующее состояние машины $\mathcal{T}_{0}$ и слово $b$, выдаваемое машиной $\mathcal{T}_{0}$ на выходную ленту. Состояние машина $\mathcal{T}$ запоминает, а слово $b$ выдает на свою выходную ленту. Далее необходимо промоделировать движения головок машины $\mathcal{T}_{0}$ по входной ленте и найти соответствующие символы, которые будут воспринимать головки машины $\mathcal{T}_{0}$ в следующий момент.

Легко рассматриваются случаи, когда движение головки не происходит либо головка переходит с одного разделительного символа 0 на соседний символ 0 (тогда данные символы 0 «ограничивают» пустое слово $z_{i}$ ). Оставшиеся случаи относятся к одному из четырех типов: головка находится внутри непустого слова и сдвигается влево (вправо), головка находится на разделительном символе 0 и сдвигается влево 
(вправо) внутрь непустого слова. По существу все четыре типа рассматриваются одинаково. Поэтому рассмотрим только первый тип (с движением головки влево).

Итак, пусть некоторая головка машины $\mathcal{T}_{0}$ расположена внутри непустого слова $z_{i}$ и сдвигается влево. Будем также предполагать, что счетчик $\mathcal{C}_{i 1}$ «фиксирует» момент времени $t$, в который машина $\mathcal{T}_{i}$ впервые выдает на выходную ленту символ $d$ слова $z_{i}$, читаемый головкой машины $\mathcal{T}_{0}$ в момент времени $t$; два остальных счетчика $\mathcal{C}_{i 2}, \mathcal{C}_{i 3}$ «содержат» число 0 . Нам необходимо определить символ, расположенный слева от символа $d$, а также момент времени, в который данный символ впервые появился на выходной ленте машины $\mathcal{T}_{i}$. С этой целью запустим процесс моделирования вычисления на машине $\mathcal{T}_{i}$, пытаясь определить тот наименьший момент времени $t^{\prime}<t$, после которого (до момента $t$ ) машина $\mathcal{T}_{i}$ выдает на выходную ленту только слово $\Lambda$. Для этого нам понадобится задействовать счетчики $\mathcal{C}_{i 2}, \mathcal{C}_{i 3}$. Именно, с помощью счетчика $\mathcal{C}_{i 2}$ мы будем последовательно «генерировать» числа $t^{\prime}$ от 1 до $t-1$ и затем моделировать работу машины $\mathcal{T}_{i}$ до момента $t$, проверяя, что после момента $t^{\prime}$ машина $\mathcal{T}_{i}$ не выдает на выходную ленту символы 1 и 2.

Более детально этот процесс выглядит так. Пусть счетчики $\mathcal{C}_{i 1}, \mathcal{C}_{i 2}$ «содержат» числа $t$ и $t^{\prime}$, где $t^{\prime}<t$, а счетчик $\mathcal{C}_{i 3}-$ число 0 . И пусть нам необходимо проверить, что после момента $t^{\prime}$ машина $\mathcal{T}_{i}$ выдает на выходную ленту только слово $\Lambda$. Начинаем моделировать вычисление на машине $\mathcal{T}_{i}$, одновременно «считая» шаги моделирования с помощью счетчика $\mathcal{C}_{i 3}$ и - в обратном порядке - с помощью счетчиков $\mathcal{C}_{i 1}, \mathcal{C}_{i 2}$. По достижении счетчиком $\mathcal{C}_{i 2}$ числа 0 продолжаем моделировать работу машины $\mathcal{T}_{i}$ и проверять пустоту/непустоту выходных слов. Кроме того, начинаем «переписывать» число $t-t^{\prime}$ со счетчика $\mathcal{C}_{i 1}$ на счетчик $\mathcal{C}_{i 2}$. В результате в любой момент времени перед достижением счетчиком $\mathcal{C}_{i 1}$ числа 0 счетчики $\mathcal{C}_{i 2}-\mathcal{C}_{i 3}$ в сумме будут давать величину $t$. Поэтому если после момента $t^{\prime}$ мы обнаружим на выходе машины $\mathcal{T}_{i}$ один из символов 1,2 , то с помощью всех трех счетчиков сумеем на одном из счетчиков зафиксировать этот момент времени, а на другом счетчике - восстановить число $t$. В этом случае для продолжения работы алгоритма необходимо дополнительно число $t^{\prime}$ перевести в число $t^{\prime}+1$. Если же после момента $t^{\prime}$ символы 1,2 на выходе не появлялись, то это значит, что в момент времени $t^{\prime}$ на выходной ленте машины $\mathcal{T}_{i}$ появился символ, непосредственно предшествующий символу $d$ (при $t^{\prime}=0$ соответствующий символ есть 0$)$.

Пусть теперь функция $g$ принадлежит классу $C$, а функция $f$ получается из функции $g$ с помощью операции ограниченной конкатенации (1). Пусть, далее, функция $g$ вычислима на машине Тьюринга $\mathcal{T}_{0}$, имеющей $k$ головок. Покажем, как определить машину Тьюринга $\mathcal{T}$, которая будет вычислять функцию $f$.

Работа машины $\mathcal{T}$ в известном смысле будет проще, чем работа машины Тьюринга, построенной для случая суперпозиции. Именно, чтобы фиксировать очередное начало $z$ слова $y$, достаточно двух головок, двигающихся только на участке входной ленты, содержащем слово $0 y 0$. Одна головка служит для обозначения конца слова $z$, другая - для сохранения числа $|z|$, когда возникает необходимость в проверке выхода одной из головок машины $\mathcal{T}_{0}$ направо со слова $z$.

Итак, машина $\mathcal{T}$ будет иметь $k+2$ головок, первые $k$ из которых точно воспроизводят положение $k$ головок машины $\mathcal{T}_{0}$ в моделируемый момент времени, а остальные две служат для выделения подслова $z$ слова $y$. Предположим поэтому, 
что в некоторый момент моделирования работы машины $\mathcal{T}_{0}$ первые $k$ головок машины $\mathcal{T}$ занимают те же положения на входной ленте, что и соответствующие головки машины $\mathcal{T}_{0},(k+1)$-я головка отмечает «границу» слова $z,(k+2)$-я головка находится на предпоследнем символе 0 , машина $\mathcal{T}$ «помнит» состояние машины $\mathcal{T}_{0}$ и для каждой из первых $k$ головок - на каком именно слове $x_{1}, \ldots, x_{n}, z$ находится данная головка. Кроме того, предполагаем, что машина $\mathcal{T}$ уже выдала на выходную ленту все значения функции $g$, отвечающие префиксам слова $z$, отличным от $z$.

Нас будет интересовать только случай, когда в результате выполнения команды машины $\mathcal{T}_{0} i$-я головка $(1 \leqslant i \leqslant k)$ сдвигается внутри слова $z$. Если она сдвигается налево либо не сдвигается, то машина $\mathcal{T}$ сразу выполняет это действие. Пусть $i$-я головка сдвигается вправо. Тогда необходимо сначала выяснить, останется ли головка в пределах слова $z$. Для этого организуем движения $i$-й и $(k+1)$-й головок влево, а $(k+2)$-й вправо с таким расчетом, чтобы, с одной стороны, проверить, находится ли $i$-я головка на последнем символе слова $z$, а с другой стороны, с помощью $(k+1)$-й и $(k+2)$-й головок сохранить число $|z|$. Если $i$-я головка находится не на последнем символе слова $z$, то возвращаем все три головки на исходные позиции и совершаем движение $i$-й головки вправо. В противном случае также возвращаемся к исходной конфигурации, но при этом «запоминаем», что $i$-я головка в следующий момент моделирования будет «воспринимать» последний символ 0 входного слова. (Если изначально $i$-я головка машины $\mathcal{T}_{0}$ находилась на последнем символе 0 , то в соответствующий момент моделирования $i$-я головка машины $\mathcal{T}$ находится на $|z|$-м символе слова $y$, но машина $\mathcal{T}$ «помнит», что $i$-я головка машины $\mathcal{T}_{0}$ воспринимает последний символ 0 . При движении $i$-й головки машины $\mathcal{T}_{0}$ влево машина $\mathcal{T}$ свою $i$-ю головку влево не сдвигает.)

При переходе от начала $z$ к непосредственно следующему началу слова $y$ машина $\mathcal{T}$ сдвигает $(k+1)$-ю головку на одну клетку вправо, а первые $k$ головок - на первый символ 0 входного слова. Теорема доказана.

Автор благодарен рецензенту за полезные замечания.

\section{Список литературы}

1. Кузнецов А.В., "К теореме о каноническорй форме для ординально-рекурсивных функций”, Приложение в кн.: Р. Л. Гудстейн, Математическая логика, Наука, Москва, $1961,149-154$.

2. Марченков С. С., Элементарные рекурсивные функиии, МЦНМО, Москва, 2003, 112 с.

3. Марченков, С. С., "Ограниченная монотонная рекурсия и МГ-автоматы”, Программирование, 2013, № $6,3-11$.

4. Grzegorczyk, A., "Some classes of recursive functions", Rozpr. Matemat., 4 (1962), 1-46.

5. Kalmar, L., "Egyszerü pelda eldönthetelen aritmetikai problemara", Mat. és fiz. lapok, 50 (1943), 1-23.

6. Skolem, Th., "Proof of some theorems on recursively enumerable sets", Notre Dame J. Formal Logic, 3:2 (1962), 65-74. 